\title{
TouchStory: Towards an Interactive Learning Environment for Helping Children with Autism to Understand Narrative
}

\author{
Megan Davis ${ }^{+}$, Kerstin Dautenhahn ${ }^{+}$, Chrystopher Nehaniv ${ }^{+}$ \\ and Stuart D. Powell* \\ ${ }^{+}$Adaptive Systems Research Group, School of Computer Science \\ *Department of Education \\ University of Hertfordshire \\ Hatfield, Hertfordshire, AL10 9AB, UK \\ corresponding author: M.Davis@herts.ac.uk
}

\begin{abstract}
Children with autism exhibit a deficit in the comprehension and creation of narrative which impacts their social world. Our ongoing research agenda is to find ways of developing interactive learning environments which enhance the ability of individual children with autism to deal with narrative and thus the social world. The study reported here involved 12 children in a longitudinal study which focussed on identifying the particular aspects of narrative which individual children found difficult. Our aim was to investigate each individual child's understanding of 'primitive' components of narrative by means of an interactive software game called TouchStory which we developed for this purpose. Our results show, for most of the children, an ongoing and clear distinction in their understanding of the various narrative components, which relates their narrative comprehension as shown by a picture-story based narrative comprehension task.
\end{abstract}

\section{Introduction}

Autism is a lifelong pervasive developmental disorder affecting social ability; people with autism exhibit impaired social interaction and communication, and have a limited range of imaginative activities, collectively referred to as the triad of impairments [1-3]. In addition, research has shown that children with autism exhibit a deficit in the comprehension and creation of narrative; particularly in understanding the motives and emotions of characters, and the reasons why events happen [4-6]. It is argued that this narrative deficit underlies the social difficulties found in autism [7-9]. Our ongoing research agenda is to find ways of developing interactive learning environments which enhance the ability of individual children with autism to deal with narrative and thus the social world. The study reported here involved 12 children in a longitudinal study which focussed on identifying the particular aspects of narrative which individual children found difficult. Our aim was to investigate each individual child's understanding of the 'primitive' components of narrative by means of an interactive software game called TouchStory which we developed for this purpose. The work falls 
under the umbrella of the Aurora project [10] which investigates the potential enhancement of the everyday lives of children with autism through the use of robots and other interactive systems as therapeutic or educational 'toys'.

\section{The Longitudinal Study: Concepts and Methodology}

Our overall aim is to enhance the abilities of children with autism to understand straightforward narrative. That is, the recounting of a sequence of events involving purposeful characters, such that there is some break in the scenario which makes the story worth telling [11]. Our aim in this study was to investigate children's understanding of primitive components of narrative. We identified these as the characters, the settings or background, and the sequence of events; further, we argue that a narrative sequence is a special form of temporal sequence therefore we include both temporal sequences and sequences with no temporal dimension which we call reversible sequences. This gives narrative and proto-narrative types as follows:

- type $c$ which addresses character variability and continuity;

- type $b$ which addresses background or setting variability and continuity;

- type $r$ which addresses the sequencing aspects of narrative in the simplified form of a reversible sequence;

- type ts which addresses temporal aspects of narrative;

- type ns which are complete mini-narratives.

We are concerned with the children's understanding of narrative per se, we are not concerned with literacy; therefore we work with picture narratives. We introduce the term t-story to mean a picture narrative or proto-narrative presented by TouchStory. T-stories were prepared for each primitive type, an example of the type $r s$ is shown in Fig. 1. The study used a set of 56 t-stories moderated for correctness and lack of ambiguity by a panel of 10 adults. The panel consisted of 7 men and 3 women, with a range of technical experience in using computers; they had no previous involvement with the project or knowledge of the children involved.

The longitudinal study took place in a day school unit for children with impaired communication. All 12 children of the unit were involved, 10 of whom, all boys, were diagnosed either with autism, or behaviours suggestive of autism. We do not claim that these children are representative of all children with autism, but consider them as individual cases, from which some generalisations may be made. Of the remaining children, both girls, one diagnosis was 'general developmental delay' and the other 'social interaction difficulties'. The children were aged between 5 and 11 years.

Twelve visits were made to the unit between February and June 2005; of these one visit was devoted to a paper-based picture-story comprehension task based on the work of Paris \& Paris [12], and one to ensuring the children realized the study was at an end. TouchStory was used on the other visits. The longitudinal study uses an adaptive phase where the stories presented by the system varied depending on the interaction history of the child, i.e. the scores that child had achieved on previous visits. The goal of this adaptation was to tailor the system towards each child's individual learn- 
ing needs, while still providing and enjoyable and rewarding experience. More details on the overall motivation for the adaptation and initial observations are described by Davis et al in [13].

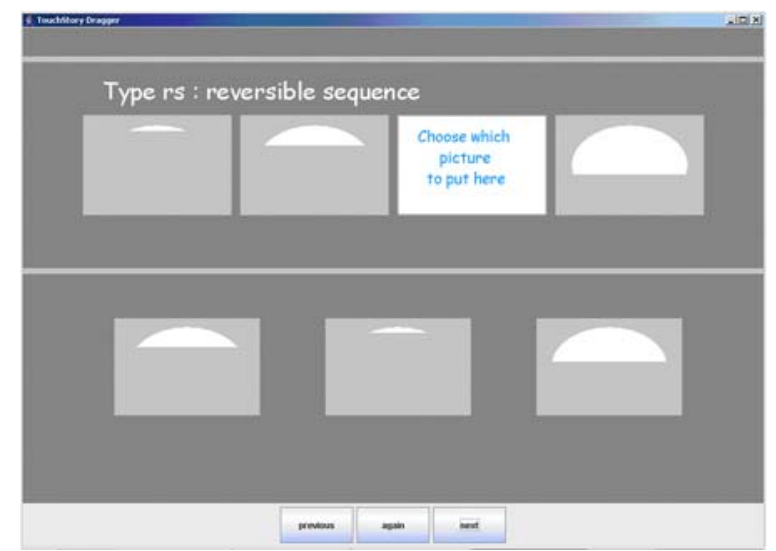

Fig. 1. TouchStory displaying a t-story from the category 'reversible sequence'

Children with autism typically have difficulty with generalising from one context to another, and with remote object references (both relevant to using a mouse), and so TouchStory was presented using a touch sensitive screen. Additionally the children prefer highly predictable environments, therefore new t-stories and new t-story types were introduced gradually as the study progressed. A typical session would consist of 12 to 14 t-stories. For each t-story the task is to select from the bottom row (see Fig.1) the most appropriate picture to complete the top row, and drag it into place. The reward for a correct answer is simply that the bottom row is removed, leaving just the completed sequence. Thus the reward re-enforces the correct answer and does not introduce extraneous sounds or effects; this we consider important in the case of software designed for children with autism as they are particularly likely to focus on seemingly irrelevant details. TouchStory allowed the children controlled autonomy during a session; for example children could use the 'next' button to move on to the next t-story, but repeated clicking of 'next' had no effect, in this way seemingly meaningless repetition, commonly observed in children with autism, was avoided.

Our approach was to incorporate TouchStory sessions into the child's school routine, in a natural, child-centred way. The emphasis throughout the study was on a playful and enjoyable context. The children's communication therapist was present during sessions and gave help or autonomy as appropriate to the individual child. Her advice on whether it was appropriate to terminate a child's session early was followed without question. This approach ensures that TouchStory sessions integrate well with the children's previous experience, that the children's immediate and changing needs are addressed, and that results obtained are relevant to later general classroom-based use of TouchStory. 


\section{$3 \quad$ Results}

The results presented here focus on the children's individual abilities within the 'primitive' narrative categories. The number of t-stories seen differs from child to child because of: the adaptive nature of the software; early termination of some sessions for some children; and absence from school, and so analysis was carried out on the proportion of t-stories answered correctly in each category. The median number of t-stories seen by a child (including repeated t-stories) was 100, and the median number of distinct t-stories seen was 45.

A cluster analysis, using single linkage Euclidian distance, of the proportion of tstories answered correctly in each category, during the study as a whole, is presented in Fig2.

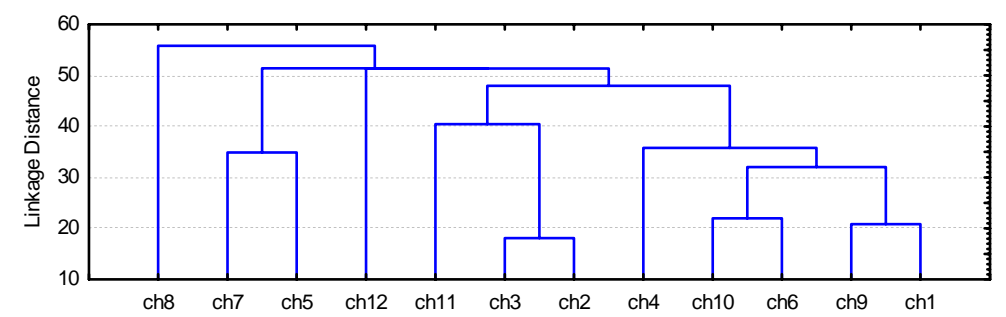

Fig. 2. Cluster analysis of the children's scores in each narrative category

This cluster analysis is used to structure the presentation of the children's understanding of the narrative components presented by TouchStory. To ground the results in activities relevant to everyday school life, results are also presented from the paperbased picture-story comprehension task (NCT), this was scored by two raters independently and the inter-rater reliability was found to be high. The maximum score available for the NCT task was 20; the score for each child is presented as a number out of 20.

The first cluster presented is the $\{\mathrm{ch} 1, \mathrm{ch} 9, \mathrm{ch} 6, \mathrm{ch} 10, \mathrm{ch} 4\}$ cluster. The children in this cluster do well with background and character t-stories and least well with the temporal sequence category. All the children in this cluster were diagnosed with autism or behaviors suggestive of autism. Table 1 presents a graph for each child in this cluster showing, for each session which the child attended, the proportion of t-stories answered correctly in each category. The graphs show a clear distinction in performance in different categories, and, in comparison with cluster 2, low variance within categories. 
Table 1. For each session attended the proportion of t-stories answered correctly in each category by each child in cluster 1

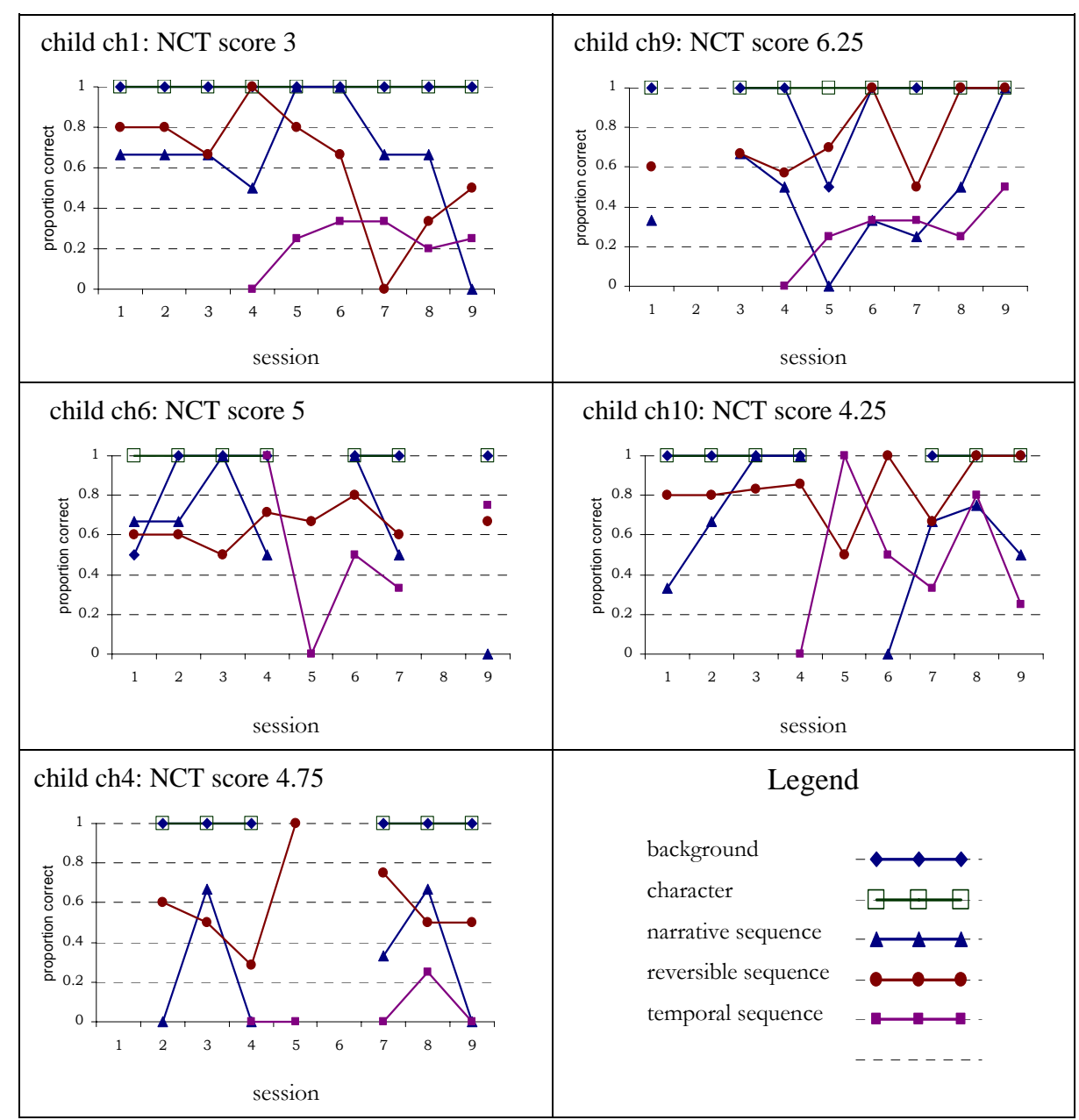

The second cluster presented is the $\{$ ch2, ch3, ch11\} cluster. Although these children were at their best with the background and character t-stories they did not have the high scores typical of children in cluster1, also, in contrast to cluster 1 , they did least well with the narrative category. Two of these children do not have diagnoses of autism; the third, at the time of the trial was diagnosed with autism, but this has recently been questioned. He certainly has other issues including dyspraxia and a short attention span. The graphs for these children can be found in Table 2. They show much greater variance within each category than the first cluster and less differentiation among categories. 
Table 2. For each session attended the proprotion of t-stories answered correctly in each category by each child in cluster 2

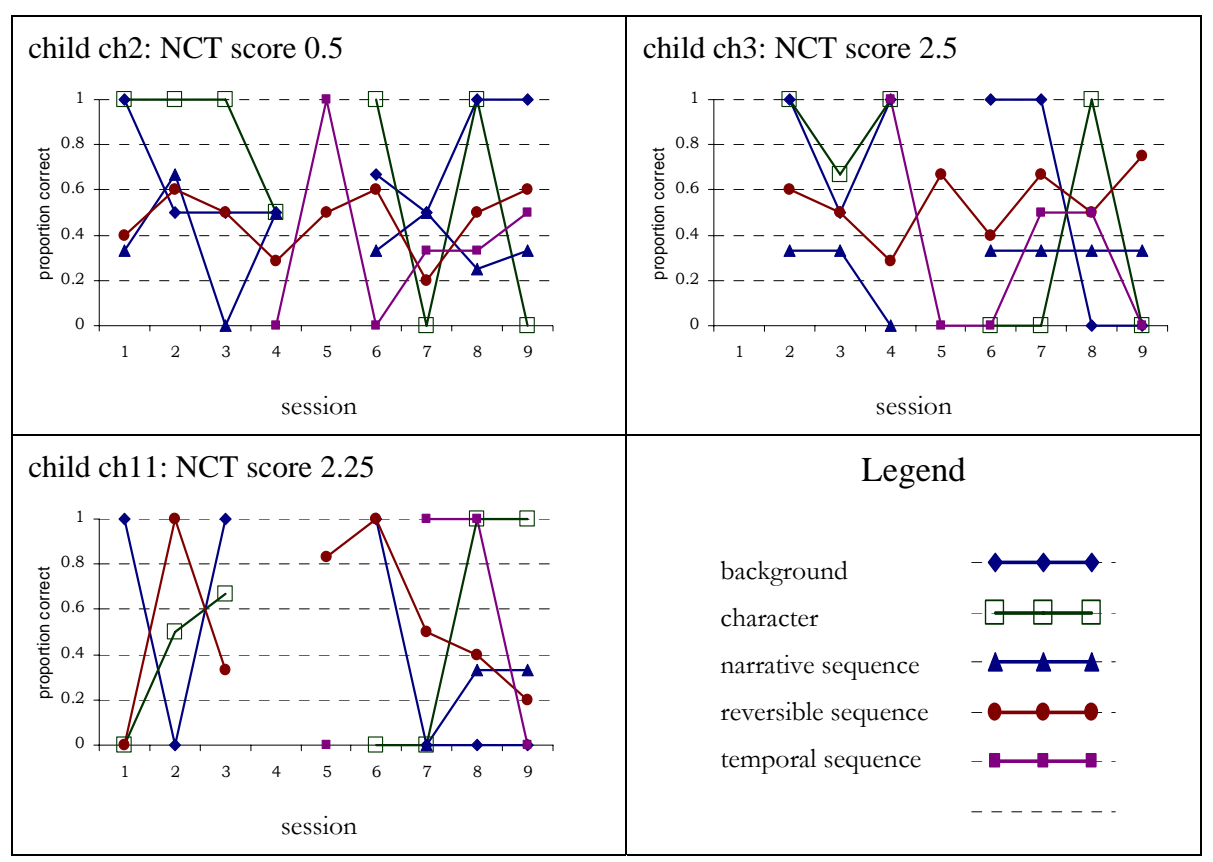

The third cluster presented is the $\{\mathrm{ch} 5, \mathrm{ch} 7\}$ cluster. These children were competent in all categories. As these children generally got the answers right, the graphs show low differentiation between categories, and low variance within them; see Table 3 . Both children had a diagnosis of autism; however child ch7 seems to have a sound grasp of narrative, he scored highly on the NCT task, he enjoys stories and his teachers find him an empathetic child. He left the unit to rejoin mainstream schooling during the study. Child ch5 did not find TouchStory trivial, he took extreme care over his answers; getting the right answer was very important to him.

Table 3. For each session attended the proportion of t-stories answered correctly in each category by each child in cluster 3 , for legend please see Table 2

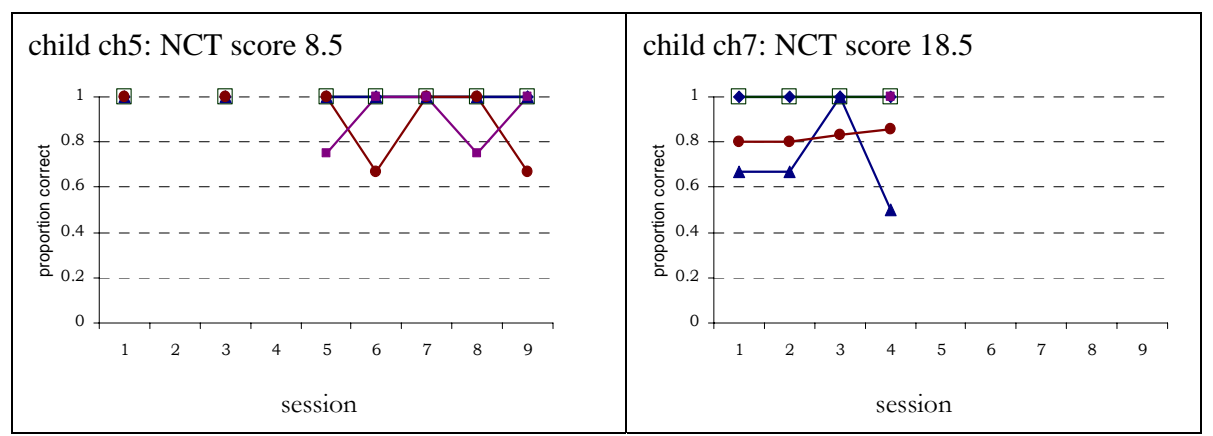


Lastly Table 4 shows graphs for the two children with results which do not cluster closely with any other. These two children are special cases; child 12 was generally unhappy throughout the study and was not interested in TouchStory, although in a previous prototype study he had been engaged and successful. Child 8 often found it difficult to focus, additionally although he was able to drag pictures across the screen he was not able to place them as the task required. In his case the first picture he moved in a definite manner was taken as his intended answer.

Table 4. For each session attended the proportion of t-stories answered correctly in each category by child 8 and child 12 . For legend please see Table 2

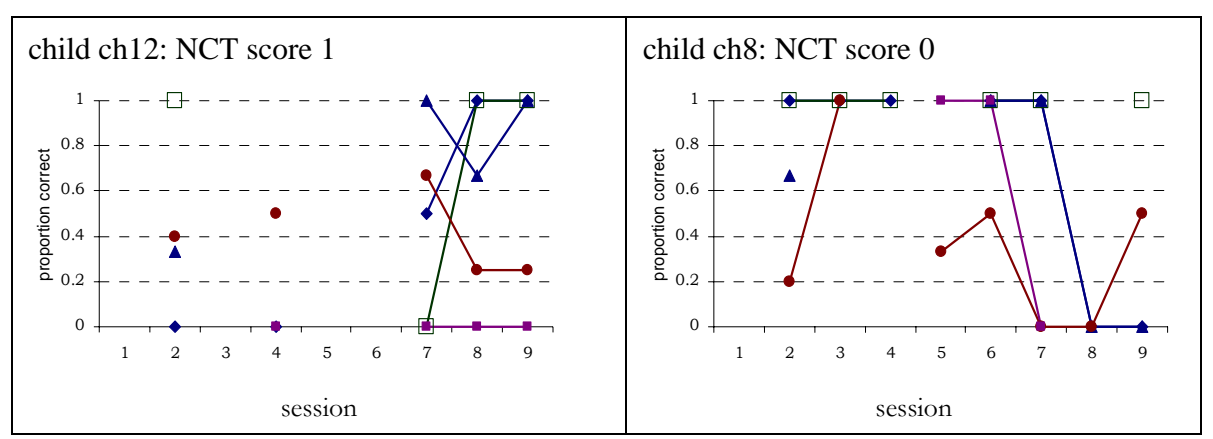

\section{Discussion}

Our results show that TouchStory can identify those aspects of narrative which individual children find difficult. Most of the children fall into three clusters; there is a correspondence between these clusters and the children's scores on the paper-based picture narrative comprehension task (NCT). The children in cluster 2 have the lowest NCT scores, in the range 0.5 to 2.5 ; those in cluster 1 are in the range 3 to 6.5 , and those in cluster 3 are in the range 8.5 to 18.5. The children in cluster 1 show the clearest differentiation among proto-narrative categories, those in cluster 3 (who are very successful with TouchStory) show a similar but compressed differentiation. The children in cluster 2 show less differentiation. Interestingly cluster 2 includes both children who have diagnoses other than autism. Note, it is not our purpose to demonstrate that children with autism differ from those without; rather we take this result as an encouraging indication that our proto-narrative approach is particularly appropriate for children with autism.

Any improvement in the abilities of individual children with autism to deal with narrative in a way that illuminates their social world can only occur over an extended elapsed time. Therefore the results presented here will inform the design of a second longitudinal study which we plan for next year. The focus of the new study will be to investigate whether children with autism can learn about narrative from an adaptive interactive system such as TouchStory in a way which improves their understanding of stories in general. In particular we note that an adaptive system - which identifies those aspects of narrative which the child finds difficult and then adapts itself to focus 
on those aspects to provide an enjoyable but challenging game - is appropriate for children such as those in cluster 1 . For children such as those in cluster 2, who present a more amorphous profile, we consider it might be more appropriate to focus on one aspect of narrative and investigate improvement in that one particular area. Although the children in cluster 3 perform similarly in the TouchStory study, their understanding of narrative differs greatly, their NCT scores differ, and their teacher's reports on their understanding of stories differ. This indicates that there are some aspects or complexities of narrative which TouchStory is not currently addressing. The next study will address these issues while preserving the emphasis on an enjoyable context.

\section{Acknowledgements}

We thank the children and staff involved in this study. Also, we thank René te Boekhorst, University of Hertfordshire, for guidance on statistical measures.

\section{References}

1. Frith, U., Explaining the Enigma. 1989: Blackwells.

2. Powell, S.D., Autism, in Developmental Psychology, D.J. Messer, Millar, S., Editor. 1999, Cambridge: Cambridge. p. 243-261.

3. Wing, L., The Autistic Spectrum: A Guide for Parents and Professionals. 1996, London: Constable.

4. Tager-Flushberg, H. and K. Sullivan, Attributing mental states to story characters: A comparison of narratives produced by autistic and mentally retarded individuals. Applied Psycholinguistics, 1995, 16: p. 241-256.

5. Capps, L., M. Losh, and C. Thurber, The Frog Ate the Bug and Made his Mouth Sad; Narrative Competence in Children with Autism. Journal of Abnormal Child Psychology, 2000. 18(2): p. 193-204.

6. Abell, F., F. Happe, and U. Frith, Do triangles play tricks? Attribution of mental states to animated shapes in normal and abnormal development. Cognitive Development, 2000 (15): p. 1-15.

7. Bruner, J. and C. Feldman, Theories of mind and the problem of autism, in Understanding other minds: perspectives from autism, S. Baron-Cohen, Editor. 1993, Oxford University Press: Oxford.

8. Dautenhahn, K., The Origins of Narrative: In Search for the Transactional Format of Narratives in Humans and Other Social Animals. International Journal of Cognition and Technology: Co-existence, Convergence, Co-evolution, 2002. 1(1): p. 97-123.

9. Hutto, D., Folk Psychological Narratives and the Case of Autism. Philosophical Papers, 2003. 32(3): p. 345-361.

10.Aurora. Aurora. 2000 [cited May 2004]; Available from: http://www.aurora-project.com.

11.Bruner, J., Actual Minds, Possible Worlds. 1986: Harvard University Press.

12.Paris, P. and P. Paris, Assessing Narrative Comprehension in Young Children. Reading Research Quarterly, 2003 38(1): p. 36-76.

13.Davis, M., et al., Towards an interactive system eliciting narrative comprehension in children with autism: A longitudinal study, in Designing Accessible Technology, P. Clarkson, P. Langdon, and P. Robinson, Eds. 2006, Springer-Verlag: London. 